\title{
ORDER PARAMETER RELAXATION IN A DILUTE BOSE GAS
}

\author{
UIrich ECKERN

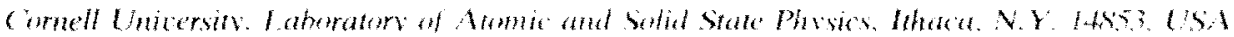 \\ and \\ Frit? HAAKE

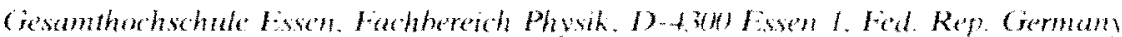

\begin{abstract}
We describe the kinetic theory of a dilute bese gas below the Bose-Einstein transition temperature, and calculate the quasiparticle scattefing time and the relaxation time of the magnitude of the order parameter. The results are important in connection with theories of the transition in spin polarized hydrogen.
\end{abstract}

Since the recent experimental stabilization of spin polarized hydrogen. attention has focused on the boson-like nature of such a fluid at low temperatures and densities [1]. In a large magnetic field. say of order 10 T. atomic hydrogen will hehave in many ways like a spin-1/2 boson. the two states being the two lowest hyperfine states. Methods to identify the superfluid statewhich might be found below $70 \mathrm{mK}$ at a density of $10^{1 / 3}$ atoms $/ \mathrm{cm}^{3}-$ as well as the hydrodynamics. have been discussed |11.

In this paper we determine one of the parameters of the hydrodynamic equations. namely the relaxation time of the magnitude of the order parameter. For simplicity. we study a system of spinless bosons. and it is realistic to consider the dilute limit: the small parameter in the seattering length. $a$, divided by the average distance between atoms. $\quad\left(\begin{array}{llll}a & 0.7 & \AA & \text { for }\end{array}\right.$ hydrogen).

We start in the usual way [2] by writing the field operators $\hat{\psi}, \hat{\psi}^{2}$ as $\hat{\psi}=\langle\hat{\psi}\rangle+\hat{\varphi}$. etc. where $\langle\dot{\psi}\rangle \equiv \psi$ is the order parameter. Assuming a point interaction of strength $\lambda$, one finds the quasiparticle energy $E_{\mathrm{p}}$ (in the simplest approximation) to

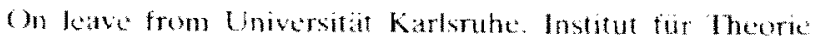
der Kondensierten Materie. D-7500 Karlsruhe 1, Ked. Ren. Gicrmans
}

he given by

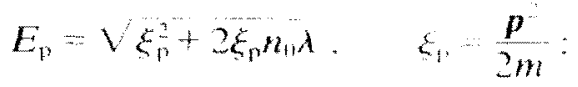

$n_{11}=\left.|\psi|\right|^{2}$ is the number of condensed particles. and $\lambda$ has to be replaced by $4 \pi^{2}{ }^{2} a / m$, in a more sophisticated theory. Since the critical temperature, $T_{2}$ is given to a good approximation by the ideal gas formula. one realizes that the linear part of the spectrum is small in the sense that

$\frac{n_{1} \lambda}{k_{k} T} \quad \frac{a}{1}(1)$

A limitation of the kinetic theory lies in the requirement that the excitation energy has to be well defined. which means that $n_{i} \lambda$ has to he large compared to the seattering rate. Since $n_{i t} \rightarrow$ 0 at $T_{\text {r. }}$, we have to exclude in the following a small region near the critical temperature, found to be given by $T_{c} \cdots T \leq T_{c}(a / l)$. This also agrees roughly with the critical region as calculated from the Ginzburg criterion.

The derivation of the kinetic theory can be performed in the same way as outlined, for example. by Aronow and Gurevich [3] in application to superconductors. The appearance of 
so-called coherence factors which describe the transformation from particles to quasiparticles is essential. For example, the total number density is given by

$n=n_{0}+\int \frac{\mathrm{d}^{3} p}{(2 \pi h)^{3}} \frac{\varepsilon_{\mathrm{p}}}{E_{\mathrm{p}}} n_{p}$

where $\varepsilon_{\mathrm{p}}=\xi_{\mathrm{p}}+n_{1} \lambda ; n_{p}$ is the distribution function of quasiparticles which obeys the usual Boltzmann equation. For the simple case under consideration:

$\dot{n}_{p}=I\left\{n_{p}\right\}$,

and $I\left\{n_{p}\right\}$ denotes the collision operator. The order parameter relaxation time can then be determined from eqs. (3) and (4), and we use a relaxation time approximation for $I\left\{n_{p}\right\}$. As could be expected, we find that the order parameter relaxation time, $\tau$, more precisely:

$$
\delta n_{0}(t) \sim \exp (-t / \tau)
$$

is given by the quasiparticle scattering time, taken for typical momenta. " Typical momenta" are determined by the weight of the integration in eq. (3), and the momentum dependence of the distribution function.) We distinguish two cases:

(I) $T / T_{\mathrm{c}} \leq a / l$, and

(II) $T / T_{\mathrm{c}} \geq a / l$.

In case (I) all scattering processes involve phonons only, and typical momenta are given by $E_{\mathrm{p}} \sim k_{\mathrm{B}} T$. The dominant term, which has already been calculated [4], is found to be:

$\mathrm{I}: \frac{\hbar}{\tau}=\left(\frac{l}{a}\right)^{5 / 2}\left(\frac{T}{T_{c}}\right)^{5} k_{\mathrm{B}} T_{\mathrm{c}}$

In the other case, II, the important momenta are determined by $E_{\mathrm{p}} \sim n_{0} \lambda$, and we estimate the relaxation time:

II: $\frac{\hbar}{\tau} \sim\left(\frac{n_{0}}{n}\right)^{1 / 2}\left(\frac{a}{l}\right)^{3 / 2}\left(\frac{T}{T_{\mathrm{c}}}\right) k_{\mathrm{B}} T_{\mathrm{c}}$,

with a numerical factor of order 10 on the righthand side of eq. (7).

Eqs. (6) and (7) are the basic result of our calculation. Inserting $a / l \sim 10^{-2}$ and the temperature dependence of $n_{i}$, one obtains:

$\tau \sim\left\{\begin{array}{l}10^{-8}\left(\frac{T_{\mathrm{c}}}{T_{\mathrm{c}}-T}\right)^{1 / 2} \frac{T_{\mathrm{c}}}{T} \mathrm{~s}, \quad 10^{-2} T_{\mathrm{c}} \leq T \leq T_{\mathrm{c}} \\ 10^{-15}\left(\frac{T_{\mathrm{c}}}{T}\right)^{5} \mathrm{~s}, \quad T \leq 10^{-2} T_{\mathrm{c}}\end{array}\right.$

with $T_{\mathrm{c}} \sim 70 \mathrm{mK}$ for the mentioned density. We want to remark that the calculation presented is in close analogy with similar calculations for superconductors [5].

\section{Acknowledgements}

We would like to thank Eric Siggia and Andrei Ruckenstein for stimulating discussions. One of us (U.E.) acknowledges a NATO fellowship through the Deutsche Akademische Austauschdienst.

\section{References}

[1] E.D. Siggia and A.E. Ruckenstein, Phys. Rev. Lett. 44 (1980) 1423; J. Phys. (Paris) 41 (1980) C7-15; Phys. Rev. B23 (1981) 3580 .

[2] P.C. Hohenberg and P.C. Martin, Ann. Phys. (N.Y.) 34 (1965) 291

[3] A.G. Aronov and V.L. Gurevich, Fiz. Tverd. Tela 16 (1974) 2656 [Sov. Phys. Solid State 16 (1975) 1722].

[4] S. Morita and F. Mohling. Phys. Rev. 120 (1960) 681.

[5] See, for example, A. Schmid. J. Phys. (Paris) 39 (1978) $\mathrm{C}(-1360$ and further references therein. 